\title{
Subkutane Infusionsanästhesie (SIA) in der operativen Dermatologie
}

\author{
Slow Infusion Tumescent Anaesthesia in Surgical Dermatology
}

Autor

Institut

\section{S. Bethge}

Klinik für Dermatologie und Allergologie, Städtische Kliniken Wiesbaden HSK, Wilhelm-Fresenius-Klinik GmbH

\section{Bibliografie}

DOI $10.1055 / \mathrm{s}-2007-966356$

Akt Dermatol 2007; 33:

168-173 @ Georg Thieme

Verlag KG Stuttgart · New York ISSN 0340-2541

Korrespondenzadresse

Dr. med. Sabine Bethge

Funktionsoberärztin

Klinik für Dermatologie und

Allergologie

Städtische Kliniken Wiesbaden

Lehrkrankenhaus der

Universität Mainz

HSK, Wilhelm-Fresenius-Klinik

$\mathrm{GmbH}$

Aukammallee 39

65191 Wiesbaden

sabine@bethge-net.de

\section{Zusammenfassung \\ $\nabla$}

Die subkutane Infusionsanästhesie ist ein modernes lokales Anästhesieverfahren, das sich in der operativen Dermatologie bewährt hat. Es ermöglicht dem Operateur, an nahezu allen Körperteilen die Verfahren der Oberflächenchirurgie

\section{Einleitung}

Die Tumeszenzlokalanästhesie (TLA) wurde von Jeffrey A. Klein Ende der 80er-Jahre des letzten Jahrhunderts zur Fettabsaugung entwickelt. Dabei wurde eine 0,04-prozentige Lidocain-Lösung mit Adrenalin-Zusatz verwendet und mehrere Liter subkutan infundiert. In Deutschland wurde die Methode von G. Sattler modifiziert und unter der Bezeichnung „Subkutane Infusionsanästhesie“ (SIA) eingeführt [1]. Er ersetzte Lidocain durch Prilocain, welches als Lokalanästhetikum vom Amid-Typ eine wesentlich geringere Systemtoxizität als Lidocain hat. Die heute vielfach verwendete SIA-Mischlösung wurde 1997 von $\mathrm{H}$. Breuninger entwickelt und enthält neben Prilocain nun auch Ropivacain als lokalanästhetisches Agens [2,3]. Ropivacain ist eine Weiterentwicklung des Lokalanästhetikums Bupivacain und zeigt geringere kardio- und zerebrotoxische Eigenschaften als letztgenanntes [4]. Es wird heute aufgrund seines Proteinbindungverhaltens vor allem bei der Periduralanästhesie in der Geburtshilfe eingesetzt. Der nicht proteingebundene Lokalanästhetikum-Anteil ist pharmakologisch aktiv und wird für das Auftreten von Toxizitätserscheinungen verantwortlich gemacht [5]. durchzuführen. Die Vorteile für den Patienten sind die geringen Nebenwirkungen dieser Methoden und die große intra- und postoperative Schmerzfreiheit. Anhand von Beispielen werden die wichtigsten Aspekte zur praktischen Durchführung (z.B. Konzentration der SIA-Lösung, Infusionsvolumen und -geschwindigkeit) erläutert.

\section{Das SIA-Verfahren \\ $\nabla$ \\ SIA-Lösung \\ Materialien}

Die Lösung setzt sich wie folgt zusammen (॰ Tab. 1):

Tab. 1 Zusammensetzung der 0,15\%igen und 0,06\%igen SIA-Mischlösung

\begin{tabular}{|lll|}
\hline & $\begin{array}{l}\mathbf{0 , 1 5 \%} \\
\text { Lösung }\end{array}$ & $\begin{array}{l}\mathbf{0 , 0 6 \%} \\
\text { Lösung }\end{array}$ \\
$\begin{array}{l}\text { Ringer-Laktat- } \\
\text { Lösung }\end{array}$ & $360 \mathrm{ml}$ & $470 \mathrm{ml}$ \\
\hline $\begin{array}{l}\text { Ropivacain } 1 \% \\
\text { Prilocain }\end{array}$ & $20 \mathrm{ml}$ & $10 \mathrm{ml}$ \\
\hline $\begin{array}{l}\text { Epinephrin } \\
\text { (Suprarenin }{ }^{\circledR} \text { ) }\end{array}$ & $20 \mathrm{ml} 2 \%$ & $20 \mathrm{ml} 1 \%$ \\
\hline $\begin{array}{l}\text { Max. Infusions- } \\
\text { volumina }\end{array}$ & $4 \mathrm{ml} / \mathrm{kg} \mathrm{KG}$ & $0,5 \mathrm{ml}$ \\
\hline
\end{tabular}

Der Adrenalinzusatz bewirkt ein deutlich blutärmeres OP-Feld und eine längere Wirkdauer der Analgesie. Jahrelange Erfahrungen an der Wiesbadener Klinik für Dermatologie und Allergologie seit 1999 mit dem Einsatz der SIA-Methode zeigten keine Adrenalin-bedingten Komplikationen. Auch Operationen an Nase und Ohren sind problemlos möglich. Lediglich an Penis und Fingern sollte die konventionelle Leitungsanästhesie zur Anwendung kommen.

Durch den Zusatz von Ropivacain ist es möglich, den Anteil des Prilocains an der SIA-Lösung zu re- 
duzieren. Bei disponierten Patienten (vor allem südländische Patienten mit Glukose-6-Phosphat-Dehydrogenase-Mangel) und höheren Prilocainmengen kann es über die Bildung von o-Toluidin zur Hemmung der Reduktion von Methämoglobin zu Hämoglobin kommen. Atemnot, Unruhe und zyanotische Verfärbung können auftreten. Ropivacain zeigt diese Nebenwirkung nicht. Es ist ein gut verträgliches, lang wirkendes Lokalanästhetikum und führt bei den Patienten zu einer postoperativ weitgehenden Schmerzfreiheit.

In jährlich ca. 2000 Operationen in der Wiesbadener Klinik für Dermatologie und Allergologie in subkutaner Infusionsanästhesie sahen wir bisher keine SIA-bedingten Komplikationen.

\section{Geräteausstattung}

Bei der SIA wird mittels Infusomat die verdünnte Lokalanästhetika-Lösung langsam und damit fast schmerzlos subkutan infundiert. Die automatische Infusion hat den Vorteil, dass die Infusionsgeschwindigkeit eingestellt werden kann und sich das Gerät nach Erreichen des vorgegebenen Volumens abstellt. Wir verwenden den Infusomat fms der Fa. B. Braun ( Abb. 1). Die Infusionsgeschwindigkeit kann in 0,1 $\mathrm{ml}$ Schritten zwischen 0,1 und 999,9 ml/h variiert werden. Das Gerät besitzt einen Luftsensor. Bei Luftblasen im Schlauchsystem stellt sich das Gerät automatisch ab und es wird ein Alarm ausgelöst. Als Schlauchsystem verwenden wir die 1 Original Infusomat ${ }^{\circledR}$-Leitung der Fa. B. Braun ggf. mit einer Heidelberger Verlängerung.

\section{Nadeln}

Die einfachste Punktion lässt sich mit einer Butterfly ${ }^{\circledR}$ Nadel erreichen. Aus unserer Erfahrung ist die Verwendung einer 25-G Butterfly ${ }^{\circledR}$ Nadel auch bei größeren Infusionsgeschwindigkeiten an jeder Körperstelle völlig ausreichend und für den Patienten schmerzarm.

\section{Technische Aspekte \\ Infusionstiefe}

Unter laufender Infusion wird die Nadel im Zentrum des zu anästhesierenden Bereichs unter das Korium ins Fettgewebe geschoben. Dieses Vorgehen ist für die Hautoberflächenchirurgie ausreichend. Bei Operationen im Bereich der Leisten und Axillen erfolgt zusätzlich eine tiefe Infiltration. Bei großen Operationsfeldern muss die Nadel gegebenenfalls noch zwei bis drei Mal umgesetzt werden. Sie wird dann schmerzfrei im Randbereich des bereits infiltrierten Areals platziert. Dieses Vorgehen erklärt die hohe Patientenzufriedenheit der SIA, da der Patient pro Operationsgebiet zumeist nur einen Stich spürt.

\section{Infusionsvolumina}

Die Infusionsvolumina variieren je nach Größe des OP-Feldes, Gewicht des Patienten und der Konzentration der SIA-Lösung zwischen 1 und $1000 \mathrm{ml}$. Nur bei großen OP-Feldern wie in ( $\triangle$ Abb.11-13) werden einige Hundert Milliliter SIA-Lösung benötigt. Fast alle Operationen an der Hautoberfläche lassen sich mit 1,0-200,0 ml SIA-Lösung ausreichend anästhesieren, sodass wir fast ausschließlich die 0,15\%ige SIA-Lösung verwenden ohne die Infusionshöchstmenge zu überschreiten.

\section{Infusionsgeschwindigkeit}

Bei der Verwendung einer 25-G Butterfly ${ }^{\circledR}$ Nadel beträgt die Infusionsgeschwindigkeit im Kopf/Hals-Bereich 50 bis $200 \mathrm{ml} / \mathrm{h}$, am Körper bis $600 \mathrm{ml} / \mathrm{h}$. Bei zu hohem Druck oder Erreichen der vorgegebenen maximalen Infusionsmenge stellt sich der Infuso-

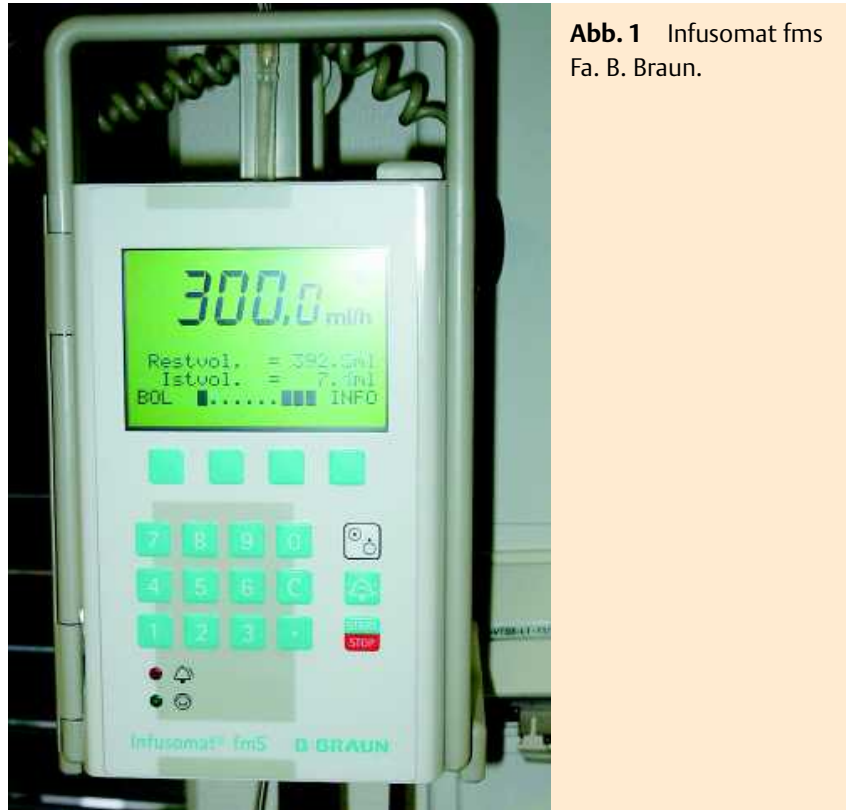

mat ab und gibt Alarm. Die o.g. Infusionsgeschwindigkeiten sind für die Patienten gut tolerierbar.

\section{Perioperatives Management \\ Wirkzeit}

Die Wartezeit bis zur ausreichenden Analgesierung beträgt bei der 0,15\%igen SIA-Lösung 10-20 Minuten, bei der 0,06\%igen Lösung ca. 30 Minuten. Durch die lange Wirkdauer der SIA ist es möglich, mehrere Patienten gleichzeitig zu anästhesieren und dann nacheinander zu operieren.

\section{Sedierung}

Beim Anlegen der SIA spürt der Patient einen oder sehr wenige Stiche und ein leichtes Druckgefühl. Ein Brennen wie bei herkömmlicher Lokalanästhesie wird durch die Verwendung von Ringer-Laktat-Lösung vermieden.

Eine Sedierung der Patienten wird bei uns nur sehr selten bei extrem ängstlichen Patienten erforderlich. In diesen seltenen Fällen ist eine Gabe von Dikaliumclorazepat (Tranxilium ${ }^{\circledR}$ ) von $10 \mathrm{mg}$ p. o. ausreichend.

\section{Überwachung}

Nach Punktion und Überwachung des Patienten bei laufender SIA für einige Minuten ist die ständige Anwesenheit eines Arztes nicht erforderlich. Die notwendige Überwachung des Patienten kann an das OP-Personal delegiert werden. Die Verantwortung bleibt beim Arzt. Die Anlage eines Pulsoxymeters zur raschen Erfassung einer Tachykardie bei versehentlicher intravasaler Infusion (Adrenalin!) ist während der gesamten Anästhesiezeit erforderlich.

\section{Indikationen \\ $\nabla$}

Die SIA kann bei sämtlichen Operationen am Hautorgan, mit Ausnahme von Penis und Fingerspitze, einschließlich aller Arten des Wundverschlusses (Lappenplastiken, Hauttransplantationen) durchgeführt werden.

Auch in der Phlebochirurgie (Stammvenen-OPs, Perforans-Dissektionen) findet die SIA ihren Einsatz [6]. 


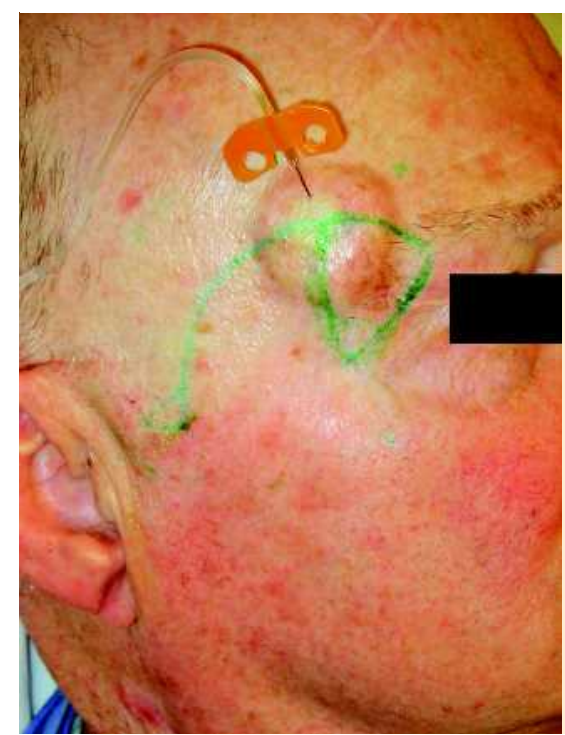

Abb. 2 Angelegte Butterfly ${ }^{\circledR}$ Nadel zur SIA bei Rotationslappenplastik an der rechten Schläfe.

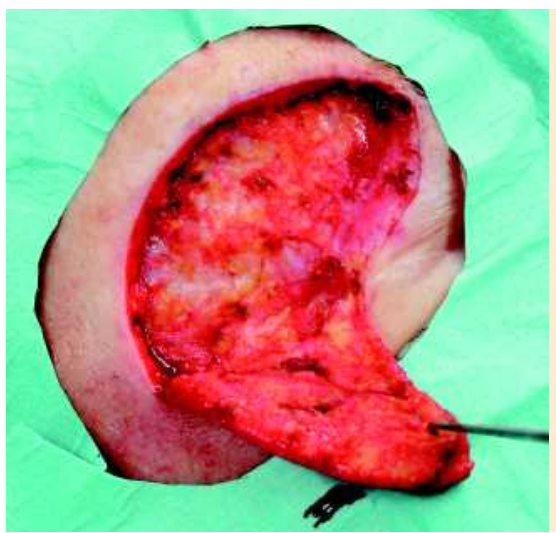

Abb. 3 Zustand nach Exzision einer Lentigo maligna und Lappenpräparation.

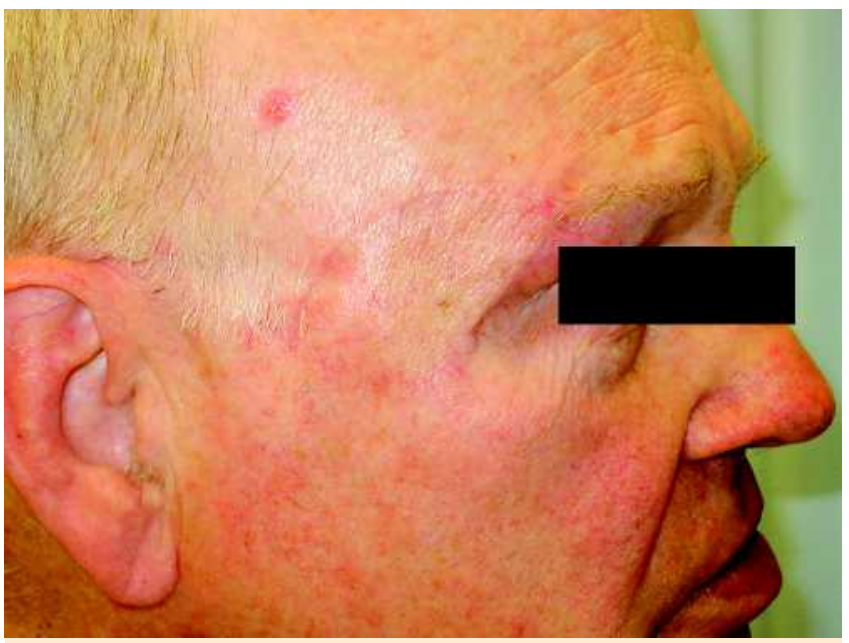

Abb. 46 Wochen postoperativ.

Bei Eingriffen in der Ulcus cruris-Chirurgie hat sich das Verfahren insbesondere beim Ulkus-Shaving bewährt.

Ein weiteres Gebiet stellen die ästhetisch-rekonstruktiven und dermato-kosmetischen Eingriffe dar.

Eine relative Indikation besteht bei der Sentinel-LymphknotenExstirpation. In unserer Hautklinik sind dies diejenigen Operationen, die mit Hilfe der Anästhesieabteilung durchgeführt werden. Natürlich lässt sich nach tiefer Infusion ein Lymphknoten sowohl in der Leiste als auch in der Axilla in SIA entnehmen. Untersuchungen zeigen jedoch, dass in ca. $25 \%$ der Fälle zusätzlich analgetische und sedierende Medikamente erforderlich waren [7]. Dies lässt sich leicht erklären, da die Sentinel-Lymphknoten oft recht tief liegen. Beim Präparieren entsteht ein unangenehmer Zug in die Tiefe.

Da wir zeitgleich mit der Sentinel-Lymphknoten-Exstirpation die Primär- oder Nachexzision des Melanoms durchführen, ist für unsere Abteilung die Intubationsnarkose das bessere und schnellere Verfahren. Spricht die Allgmeinsituation des Patienten gegen eine Vollnarkose, ist die SIA eine mögliche Alternative.

\section{Wirtschaftliche Rahmenbedingungen $\nabla$}

In der heutigen Zeit ist vor allem die Wirtschaftlichkeit ein gewichtiges Argument für eine Methode im Gesundheitswesen. Hier bietet die SIA eine ganze Reihe von Vorteilen gegenüber den alternativen Anästhesieverfahren. Kleinere Exzisionen (z. B. Naevi) können weiterhin in üblicher Lokalanästhesie durchgeführt werden. Aufgrund der Materialkosten ist hier die SIA teurer, jedoch hat man eine Zeit- (und damit auch Geld-) Ersparnis, wenn die Betäubung nicht auf dem OP-Tisch vom Operateur durchgeführt werden muss. Bei größeren Operationen, die unter Inanspruchnahme der Anästhesieabteilung durchgeführt werden müssten, ist der Vorteil der SIA wesentlich größer. Die Personalkosten Anästhesist/Anästhesiepflege entfallen und die Materialkosten der SIA gegenüber einer Intubationsnarkose/Regionalanästhesie sind deutlich geringer. Auch die Unabhängigkeit von einem Narkosearzt bietet Zeit- und Organisationsvorteile. Darüber hinaus wird in unserer Hautklinik in einem separaten Raum bei drei Patienten gleichzeitig die SIA angelegt. Die Operationen erfolgen dann nacheinander (bis zu 15 Patienten an einem vollen Saaltag), was zu einer optimalen OP-Ausnutzung führt. Durch die SIA können somit pro Zeiteinheit mehr Patienten operiert, d.h. vorhandene Ressourcen besser genutzt werden.

\section{Vor- und Nachteile $\nabla$ \\ Vorteile}

Die SIA ist gut verträglich und nebenwirkungsarm. Sie lässt sich schmerzarm anlegen (1-3 Stiche) und zeigt im Gegensatz zur üblichen Lokalanästhesie kein „Brennen“. Die lange Analgesiedauer bietet für den Patienten eine weitgehende postoperative Schmerzfreiheit. Daraus resultiert die gute Compliance der Patienten [8]. Der Adrenalinzusatz ermöglicht ein blutarmes und damit übersichtliches Operieren. Durch unterschiedliche Konzentrationen ist mit der verdünnten SIA-Lösung auch eine Operation mit großem OP-Feld möglich. Wenn ein spezieller SIARaum mit mehreren Liegen vorhanden ist, lassen sich mehrere Patienten parallel anästhesieren, die dann ohne Wartezeiten nacheinander vom Operateur versorgt werden. Dies führt zu einer besseren Personal- und Raumauslastung und spart Kosten. Die Vorteile der Unabhängigkeit von der Anästhesieabteilung wurden oben beschrieben.

\section{Nachteile}

Es gibt immer wieder Autoren, die schwere Zwischenfälle unter TLA bei Fettabsaugungen beschreiben, allerdings handelt es sich dabei um Patienten, die große Volumina erhielten. Zwischenfäl- 
le treten auch bei versehentlicher intravasaler Gabe größerer Mengen von SIA-Lösung auf [9].

Zur Überwachung des Patienten ist daher wie erwähnt die Anlage eines Pulsoxymeters vor Beginn der SIA erforderlich, um anhand einer auftretenden Tachykardie eine intravasale Infusion rasch zu erkennen.

Manche Operateure beschreiben das „feuchte“ OP-Feld als Nachteil der SIA. Nach einer kurzen Gewöhnungsphase ist dies unsererseits eher als Vorteil zu sehen, da die Gewebestrukturen auseinanderweichen, sich deutlicher darstellen und somit leichter zu präparieren sind.

Den oft beschriebenen Nachteil für den Operateur, dass der Patient wach ist, kann man auch positiv sehen. Während der einlaufenden SIA kann falls nötig beruhigend auf den Patienten eingegangen werden, und während der Operation lässt sich das Arzt/Patient-Verhältnis durch ein persönliches Gespräch festigen.

\section{Praktische Beispiele}

$\nabla$

\section{Schläfenregion}

Bei diesem Beispiel handelt es sich um einen 73-jährigen männlichen Patienten mit einer Lentigo maligna im Bereich der rechten Schläfe. Die SIA erfolgte mit $54 \mathrm{ml}$ einer 0,15-prozentigen SIA-Lösung. Die Applikation erfolgte mit einer Flussgeschwindigkeit von $150 \mathrm{ml} / \mathrm{h}$ über eine 25-G Butterfly ${ }^{\circledR}$ Nadel. Nach 20 Minuten Wartezeit konnte die Exzision mit anschließender Rotationslappenplastik durchgeführt werden.

Tipp für die Praxis: Die Anzeichnung der Arteria temporalis sollte vor Anlegen der SIA erfolgen( $\odot$ Abb. 2-4).

\section{Unterlippe}

Bei diesem 70 Jahre alten männlichen Patienten wurde im Bereich der Unterlippe ein spinozelluläres Karzinom exzidiert und die Wunde mittels W-Plastik verschlossen. Menge SIA-Lösung: $43 \mathrm{ml}$, Konzentration: 0,15\%, Flussgeschwindigkeit: $200 \mathrm{ml} / \mathrm{h}$, Wartezeit: 15 Minuten. Trotz penetrierendem Schnitt mit Resektion der bukkalen Schleimhaut reicht es, die Infusionsnadel von außen tief unter das Korium zu platzieren( $\odot$ Abb. 5-7).

\section{Ohrmuschel}

Bei diesem 72-jährigen Patienten wurde die Nachexzision eines nodulären malignen Melanoms (Tumordicke $4,5 \mathrm{~mm}$ ) mit einem Sicherheitsabstand von einem Zentimeter in Form einer dreifach keilförmigen Chondrektomie nach Trendelenburg durchgeführt. Die SIA erfolgte mit einer 0,15\%igen Lösung. Flussgeschwindigkeit: $50 \mathrm{ml} / \mathrm{h}$, Menge SIA-Lösung: $11 \mathrm{ml}$, Wartezeit: $15 \mathrm{~min}$. In diesem Fall muss die Nadel von beiden Seiten des Ohrknorpels platziert werden $(\odot$ Abb. 8-10).

\section{Rücken}

Hier handelt es sich um ein noduläres malignes Melanom mit einer Tumordicke von 3,2 mm auf Höhe Th 9 über den Dornfortsätzen. Bei diesem 64-jährigen Patienten erfolgte die Exzision mit zwei Zentimetern Sicherheitsabstand und einem Wundverschluss mittels Rotationsverschiebung nach Webster. Menge SIA-Lösung: $630 \mathrm{ml}$, Konzentration: 0,06\%, Flussgeschwindigkeit: $500 \mathrm{ml} / \mathrm{h}$, Wartezeit: $40 \mathrm{~min}$.

Wegen der Größe des OP-Feldes wurde hier die SIA-Lösung über zwei Infusomaten parallel infudiert ( $\odot$ Abb. 11-13).

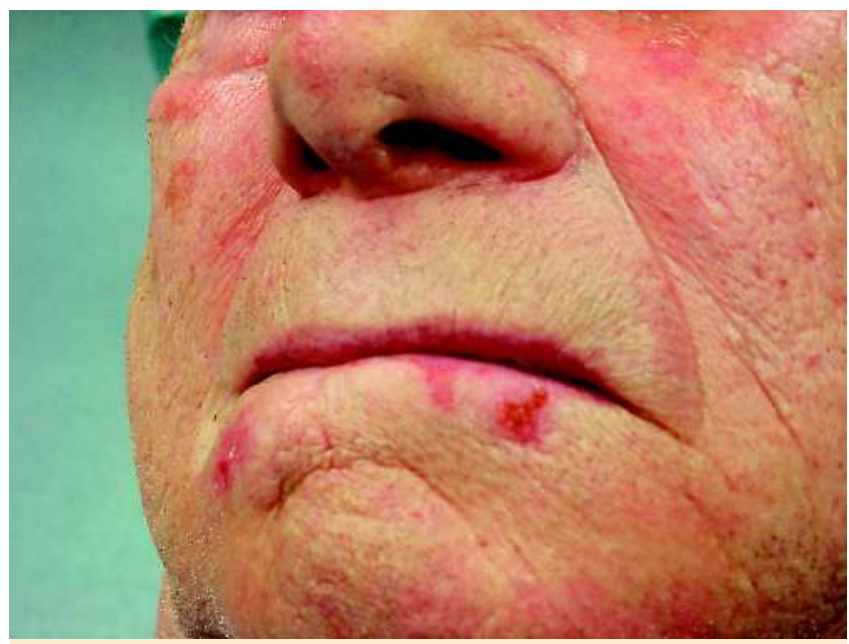

Abb. 5 Histologisch gesichertes spinozelluläres Karzinom an der Unterlippe.

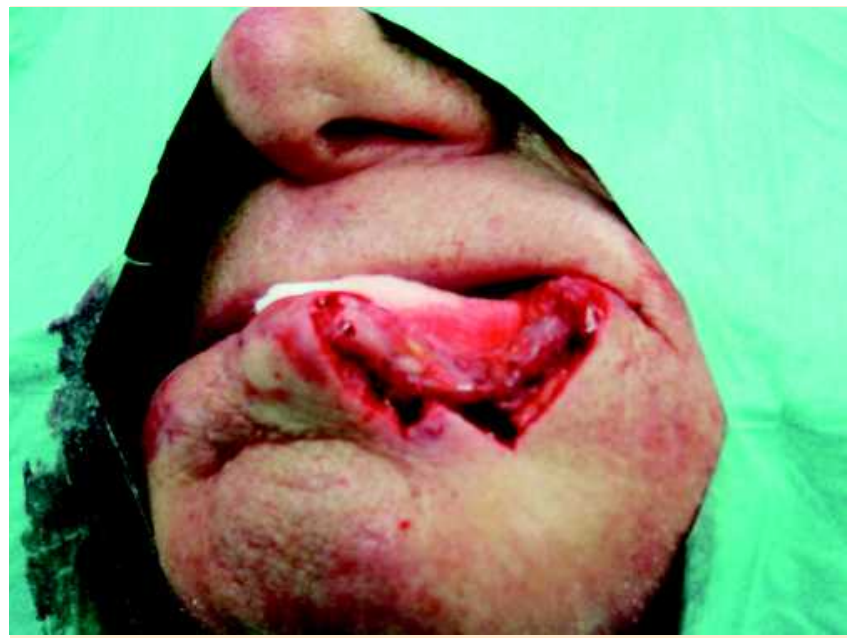

Abb. 6 W-förmige Exzision des spinozellulären Karzinoms.

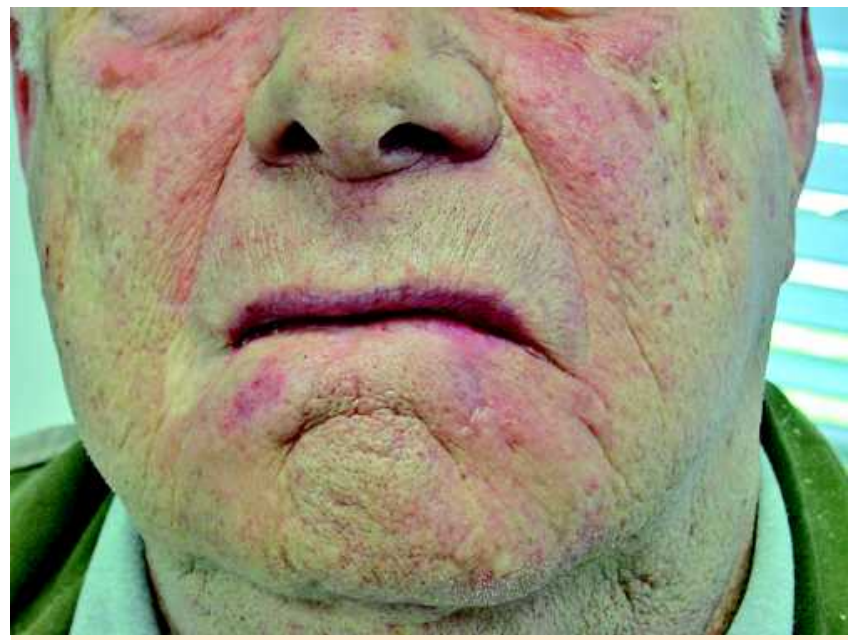

Abb. 74 Wochen postoperativ nach W-Plastik an der Unterlippe. 


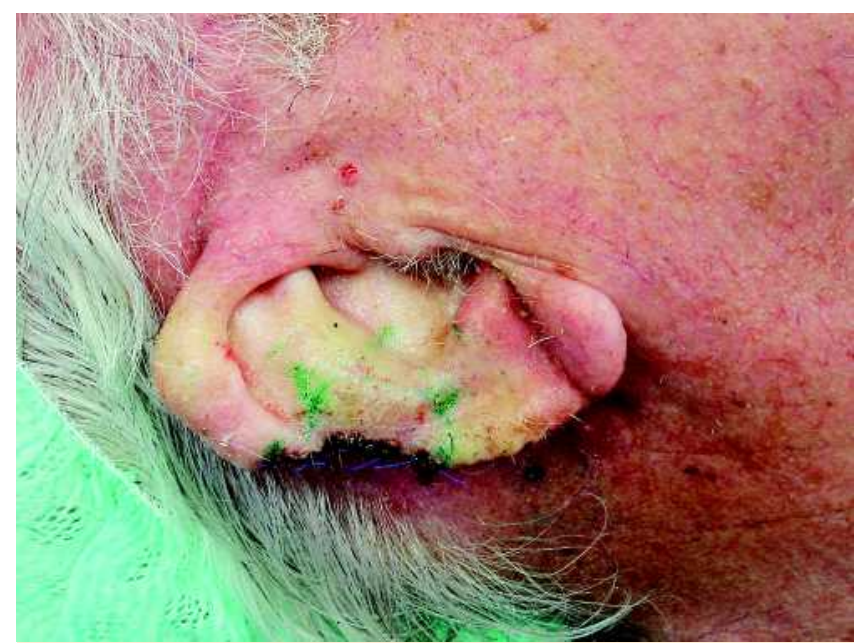

Abb. 8 Angelegte SIA zur Nachexzision bei Z.n. Exzision eines nodulären malignen Melanoms an der rechten Ohrmuschel.

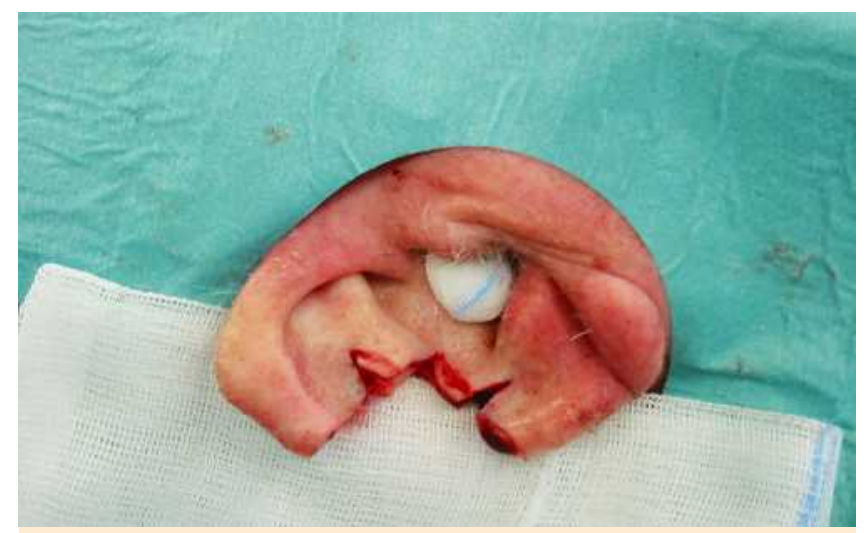

Abb. 9 Nachexzision mittels dreifach keilförmiger Chondrektomie.

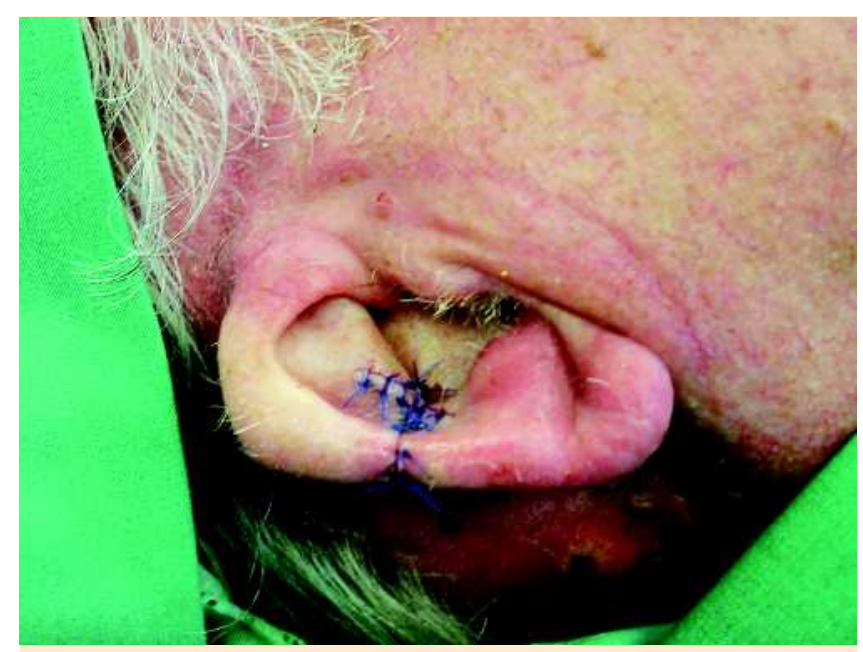

Abb. 10 Mehrschichtiger Wundverschluss am Ohr.

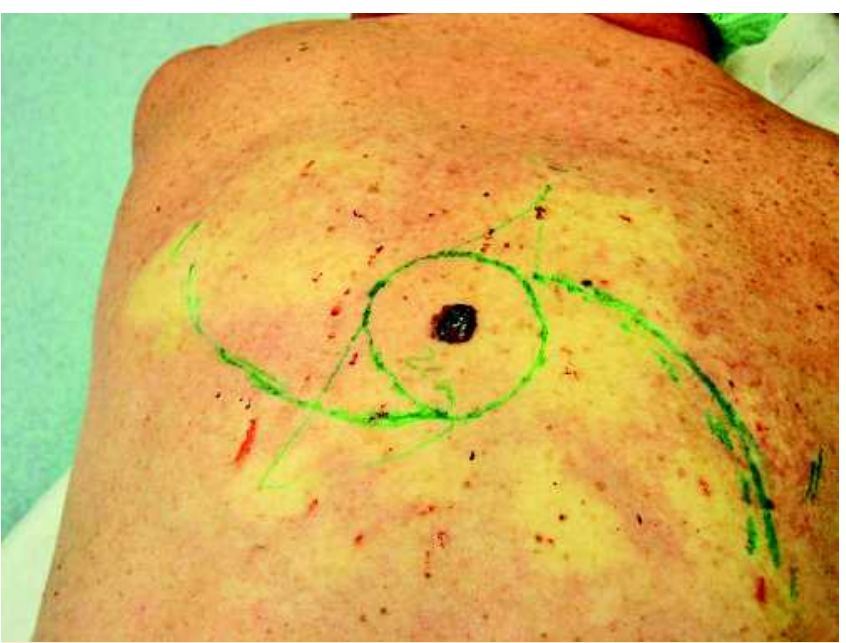

Abb. 11 Angelegte SIA zur Exzision eines nodulären malignen Melanoms mit $2 \mathrm{~cm}$ Sicherheitsabstand am Rücken.

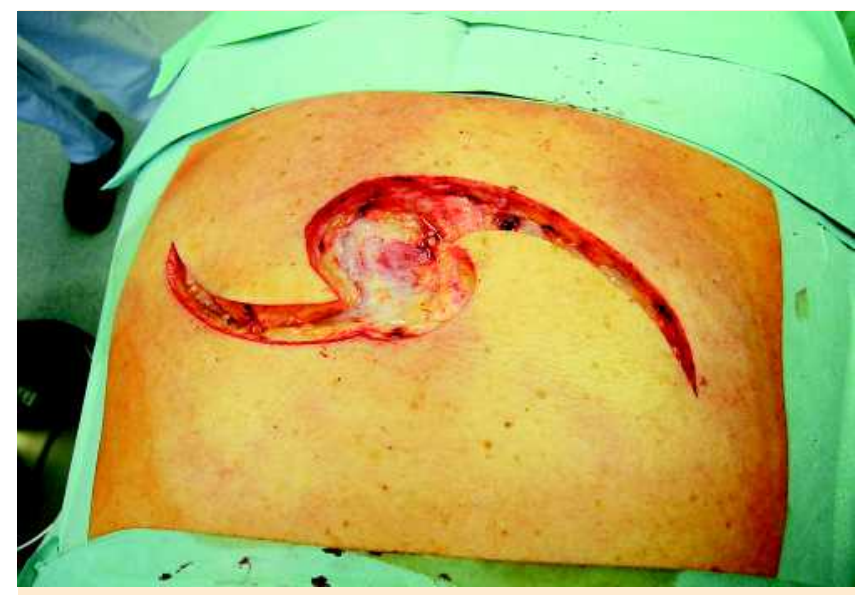

Abb. 12 Intraoperativer Zustand nach Exzision des Melanoms und Präparation der Rotationslappen.

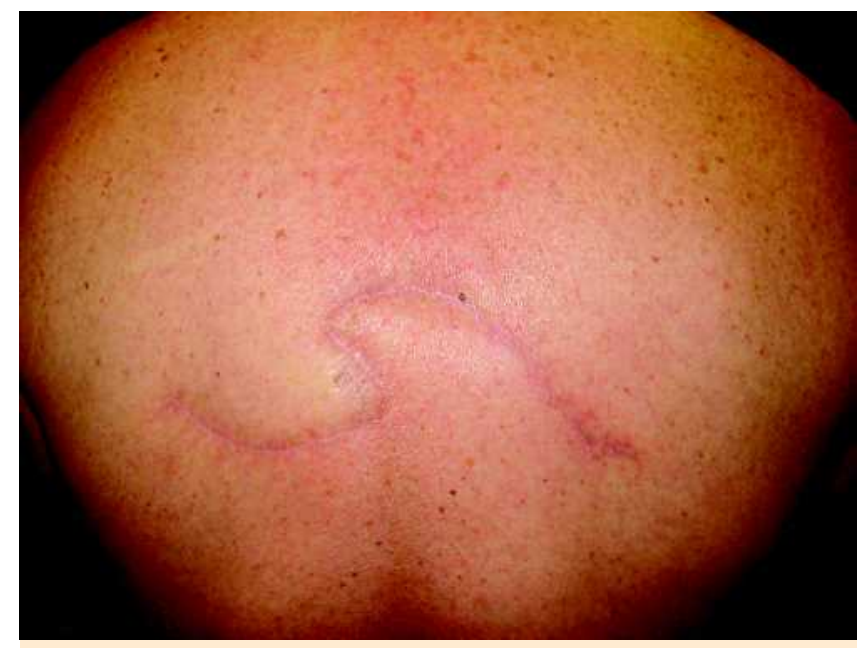

Abb. 136 Wochen postoperativ. 
Fazit

$\nabla$

Aus unserer mehrjährigen Erfahrung mit der SIA bietet dieses Narkoseverfahren überwiegend Vorteile für den Patienten und den Operateur.

Sie lässt sich bei fast allen in Hautkliniken durchgeführten Operationen anwenden und schafft eine Unabhängigkeit von der Anästhesieabteilung.

Die wirtschaftlichen Rahmenbedingungen, unter denen die stationäre Dermatologie im Bereich gesetzlich versicherter Patienten in den nächsten Jahren betrieben werden muss, werden immer enger. Dies gilt insbesondere für den Bereich der operativen Leistungserbringung mit seinem fließenden Übergang in den teilstationären und ambulanten Bereich. Effiziente Abläufe, geringe Personal- und Materialkosten und hohe Patientenzufriedenheit werden eine zunehmende Bedeutung erhalten. Die SIA leistet hierzu einen großen Beitrag.

\section{Abstract}

\section{Slow Infusion Tumescent Anaesthesia in Surgical Dermatology \\ $\nabla$}

The slow infusion tumescent anaesthesia has proved itself in the surgical dermatology. It enables the surgeon in nearly all body parts to carry out the procedures of the surface surgery. The advantages for the patient are the low side effects of these methods and intra- and post-surgical painlessness. On the basis of examples, the most important aspects are explained for the practical realisation (e.g., concentration of the anaesthesia solution, given volume and drip speed).

\section{Literatur}

1 Sommer B, Sattler G. Tumeszenzlokalanästhesie. Weiterentwicklung der Lokalanästhesieverfahren für die operative Dermatologie. Hautarzt 1998; 49: $351-360$

2 Breuninger H, Nogova L, Hobbach PS, Schimek F. Ropivacain, ein vorteilhaftes Anästhetikum für die subkutane Infusionsanästhesie (SIA). Hautarzt 2000; 51: 759-762

3 Breuninger $H$, Wehner-Caroli J. Subkutane Infusionsanästhesie (SIA) mit durch Ringer-Lösung verdünntem Prilocain. Hautarzt 1998; 49: $709-713$

4 Feldman HS, Arthur GR, Covino BG. Comparative systemic toxicity of convulsant and supraconvulsant doses of intravenous ropivacaine, bupivacaine and lidocaine in the conscious dogs. Anesth Analg 1989; 69: 794

5 Bethge S. Proteinbindung von Bupivacain und Ropivacain bei Schwangeren, Nicht-Schwangeren und Neugeborenen. Gießen: InauguralDissertation, 1993

6 Proebstle TM, Bethge S, Barnstedt S, Kargl A, Knop J, Sattler G. Subfascial endoscopic perforator surgery with tumescent local anesthesia. Dermatol Surg 2002; 28: 689-693

7 Eichhorn K, Renner R, Frithiof Haustein $U$. Sentinel node biopsy in melanoma of the trunk and the extremities in tumescent local anesthesia. Dermatol Surg 2004; 30: 253-256

8 Namias A, Kaplan B. Tumescent anesthesia for dermatologic surgery. Cosmetic and noncosmetic procedures. Dermatol Surg 1998; 24 : $755-758$

9 Beck-Schimmer B, Pasch T. Tumescent technique for local anesthesia. Anästhesiol Intensivmed Notfallmed Schmerzther 2002; 37: 84-88 